\title{
Possibility of Deconfined Criticality in SU(N) Heisenberg Models at Small $N$
}

\section{$\operatorname{AUTHOR}(\mathrm{S})$ :}

Harada, Kenji; Suzuki, Takafumi; Okubo, Tsuyoshi; Matsuo, Haruhiko; Lou, Jie; Watanabe, Hiroshi; Todo, Synge; Kawashima, Naoki

\section{CITATION:}

Harada, Kenji ...[et al]. Possibility of Deconfined Criticality in SU(N) Heisenberg Models at Small N. Physical Review B 2013, 88(22): 220408(R).

ISSUE DATE:

2013-12-13

URL:

http://hdl.handle.net/2433/200790

RIGHT:

(C)2013 American Physical Society 


\title{
Possibility of deconfined criticality in $\mathrm{SU}(N)$ Heisenberg models at small $N$
}

\author{
Kenji Harada, ${ }^{1}$ Takafumi Suzuki, ${ }^{2}$ Tsuyoshi Okubo, ${ }^{3}$ Haruhiko Matsuo, ${ }^{4}$ Jie Lou, ${ }^{5}$ Hiroshi Watanabe, ${ }^{3}$ \\ Synge Todo, ${ }^{3}$ and Naoki Kawashima ${ }^{3}$ \\ ${ }^{1}$ Graduate School of Informatics, Kyoto University, Kyoto 615-8063, Japan \\ ${ }^{2}$ Graduate School of Engineering, University of Hyogo, Himeji 671-2280, Japan \\ ${ }^{3}$ Institute for Solid State Physics, University of Tokyo, Kashiwa 5-1-5, Chiba 277-8581, Japan \\ ${ }^{4}$ Research Organization for Information Science and Technology, Kobe 650-0047, Japan \\ ${ }^{5}$ Department of Physics, Fudan University, Shanghai 200433, China
}

(Received 27 June 2013; revised manuscript received 24 September 2013; published 13 December 2013)

\begin{abstract}
To examine the validity of the scenario of the deconfined critical phenomena, we carry out a quantum Monte Carlo simulation for the $\mathrm{SU}(N)$ generalization of the Heisenberg model with four-body and six-body interactions. The quantum phase transition between the $\mathrm{SU}(N)$ Néel and valence-bond solid phases is characterized for $N=2$, 3 , and 4 on the square and honeycomb lattices. While finite-size scaling analysis works well up to the maximum lattice size $(L=256)$ and indicates the continuous nature of the phase transition, a clear systematic change towards the first-order transition is observed in the estimates of the critical exponent $y \equiv 1 / v$ as the system size increases. We also confirm the relevance of a squared valence-bond solid field $\Psi^{2}$ for the SU(3) model.
\end{abstract}

DOI: 10.1103/PhysRevB.88.220408

PACS number(s): 75.10.Jm, 05.30.Rt, 75.40.Cx, 75.40.Mg

In this Rapid Communication, we consider a quantum phase transition that is presumably described by the scenario of the deconfined critical phenomena (DCP). ${ }^{1-3}$ The DCP transition takes place between the Néel state and the valence-bond solid (VBS) state in two dimensions. It is remarkable that the symmetry group of neither phase is a subgroup of the other. This novel property is an outcome of the intrinsic lattice symmetry and an effect of the Berry phase of which no direct counterpart exists in classical critical phenomena. It was also argued that such a phase transition is realized in some frustrated magnets ${ }^{4}$ as well as superconductors. ${ }^{5}$ The Heisenberg model generalized to the $\mathrm{SU}(N)$ symmetry is a candidate for a minimal model that realizes such a quantum criticality. In the square lattice case, while its ground state for $N \leqslant 4$ is the $\operatorname{SU}(N)$ Néel state, for $N \geqslant 5$ it is the columnar VBS state. ${ }^{6-8}$ Interestingly, the VBS ground state of the $\mathrm{SU}(N)$ Heisenberg model with $N \geqslant 5$ shows an approximate $U(1)$ degeneracy, ${ }^{9}$ suggesting that the model is close to the critical point described by the DCP scenario. In the square lattice $J-Q$ model that includes an additional four-body spin interaction, ${ }^{10}$ one can take the model to its exact transition point by tuning the amplitude of the new term. The quantum Monte Carlo (QMC) results of the $\mathrm{SU}(2) J-Q$ model on the square lattice $\mathrm{e}^{10-12}$ showed a good finite-size scaling (FSS), indicating the critical nature of the transition. Another set of QMC studies ${ }^{13}$ not only showed good FSS plots, but also some universal properties shared by two models with different multibody interaction terms. Based on these and additional numerical results for $N \geqslant 5$, it was pointed out ${ }^{14}$ that the $N$ dependence of the critical indices is consistent with the $1 / N$ expansion. To examine more directly the effect of the Berry phase, which is crucial to the DCP scenario, the bilayer $\mathrm{SU}(N)$ Heisenberg model was studied, ${ }^{15}$ and the results showed that the Néel-VBS quantum phase transition for $N \geqslant 5$ becomes clearly of the first order upon introduction of the interlayer couplings. However, a numerical work on the $(2+1)$-dimensional noncompact $\mathrm{CP}^{1}$ model, ${ }^{16}$ which is believed to belong to the same universality class, suggested that the transition could be accompanied by a very small but finite discontinuity. The $\mathrm{SU}(2) J-Q$ model on the square lattice was directly compared ${ }^{17}$ with the noncompact $\mathrm{CP}^{1}$ model. It showed that there is a range of length scale in which the two models have the same behavior, while they depart from each other above a certain length scale. It was argued that the deviation indicates the nonuniversality of the transition, perhaps a first-order nature, although the possibility of another fixed point was not completely ruled out.

With all the arguments and evidence, the issue has not been completely settled. Below we present results of a systematic study of the $\mathrm{SU}(N) J-Q$ model obtained with a large scale QMC simulation. Namely, we have carried out QMC simulations on the SU(2), SU(3), and SU(4) models not only on the square lattice but also on the honeycomb lattice. We have also extended the system size ranges up to $L=256$ and 96 in the square and the honeycomb lattices, respectively. Our results do not put an end to the controversy, but instead they show (i) apparent universal critical behaviors shared by these lattice models within a limited size range up to $L \sim 96$, (ii) a clear systematic change towards the first-order transition in the estimates of critical indices as a function of the system size in the SU(2) and SU(3) cases, and (iii) relevance of the $\Psi^{2}$ scaling field in the SU(3) case with the assumption of the criticality.

Model. The $\operatorname{SU}(N) J-Q$ Heisenberg model on the square lattice can be expressed most simply in terms of the projector to the color-singlet state $P_{i j}$ :

$$
H=-J \sum_{(i j)} P_{i j}-Q \sum_{(i j)(k l)} P_{i j} P_{k l},
$$

where the summation in the second term runs over all pairs of bonds nearest neighbor and parallel to each other. Namely, the summation over $(i j)(k l)$ includes two kinds of dimer coverings of each unit square [Fig. 1(a)]. Likewise, the model on the honeycomb lattice is defined by including the six-body interaction instead of four, i.e., $-Q \sum_{(i j)(k l)(m n)} P_{i j} P_{k l} P_{m n}$ for all unit hexagons, and the summation over $(i j)(k l)(m n)$ includes two kinds of dimer coverings for each hexagon 
(a)

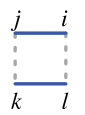

(b)

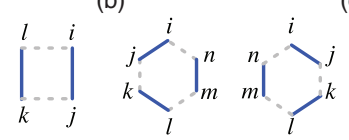

(c)

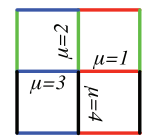

(d)

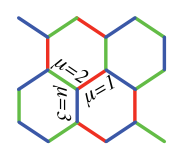

FIG. 1. (Color online) The dimer coverings of (a) unit square and (b) unit hexagon in $Q$ terms and the classification of the bonds in the definition of the complex order parameter $\Psi_{r}$, characterizing the VBS phase on (c) the square and (d) the honeycomb lattice.

[Fig. 1(b)]. Thus the $Q$ term does not break the lattice rotational symmetry in either case. The color-singlet projector is expressed in terms of the generators of the $\mathrm{SU}(N)$ algebra as $P_{i j}=-\frac{1}{N} \sum_{\alpha=1}^{N} \sum_{\beta=1}^{N} S_{i}^{\alpha \beta} S_{j}^{\beta \alpha}$. We adapt the fundamental representation on one sublattice and the conjugate representation on the other sublattice. Our QMC simulation is based on the world-line representation with the loop update ${ }^{18}$ and its generalization to the $\mathrm{SU}(N)$ model. ${ }^{8}$ We used the modified ALPS/LOOPER code ${ }^{19,20}$ with parallelization. ${ }^{21}$ In approaching the quantum critical point, we set the inverse temperature $\beta$ as $\beta J=L$, with the periodic square (rhombic) boundary condition on the square (honeycomb) lattice. In the FSS analysis, we used the Bayesian method ${ }^{22}$ for obtaining the parameters.

Scaling of the magnetic order parameter. We define the "magnetic order parameter" as $m \equiv \frac{1}{V} \sum_{i}\left(S_{i}^{\alpha \alpha}-1 / N\right)=$ $\frac{1}{V} \sum_{i} m_{i, \alpha}$, with $V$ being the total number of sites, which agrees with the staggered magnetization in the $\mathrm{SU}(2)$ case. We try to fit the obtained estimates of the magnetization to the FSS form $\left\langle m^{2}\right\rangle=L^{-2 x_{m}} \tilde{\chi}\left(t L^{y}\right)$ with $t \equiv q-q_{c}$ and $y \equiv 1 / \nu$, where $q \equiv Q /(J+Q)$. Figure 2 shows the result of the fitting with limited system sizes $L \leqslant 96$ in the case of the $\mathrm{SU}(3)$ model on both the square and honeycomb lattices. (This system-size range is simply due to the fact that the largest system on the honeycomb lattice studied here is $L=96$.) The values used for Fig. 2 are $q_{c}=0.3353$ for the square lattice and $q_{c}=0.2036$ for the honeycomb lattice. The same values of exponents $y=1.87$ and $2 x_{m}=1+\eta_{m}=1.40$ are used for both lattices. The horizontal and vertical axes for the honeycomb lattice are rescaled to match those of the square lattice. Note that not only do different system sizes fall on the same curve, but also two lattices with different lattice rotational symmetries also collapse on the same curve. Since the symmetry that is broken at the transition point is different in the two cases, a different universality class may be naturally expected. Therefore, this universal behavior supports the DCP scenario. From a similar analysis we obtain reasonably good FSS plots for SU(2) and SU(4) models as well. The data for the SU(2) model produce the best fitting with $y=1.78$ and $2 x_{m}=1.289$ at $q_{c}=0.9585$ and $q_{c}=0.5440$ for the square and the honeycomb lattice, respectively. Note here that we use data for $L \leqslant 128$ and 96 for the square and honeycomb lattices, respectively. These values are consistent with a recent parallel work that reports the DCP on the SU(2) honeycomb lattice model. ${ }^{23}$ For the data for the SU(4) model with $L \leqslant 96$, the best fitting is obtained with $y=1.59$ and $2 x_{m}=1.486$ at $q_{c}=0.0829$ and $q_{c}=0.0154$ for the square and the honeycomb lattice, respectively. The inset of Fig. 2 shows the two-point correlators $C_{m}\left(R_{i j}\right) \equiv \frac{1}{N} \sum_{\alpha}\left\langle m_{i, \alpha} m_{j, \alpha}\right\rangle$ as a function of the distance for the $\mathrm{SU}(3)$ models on the square

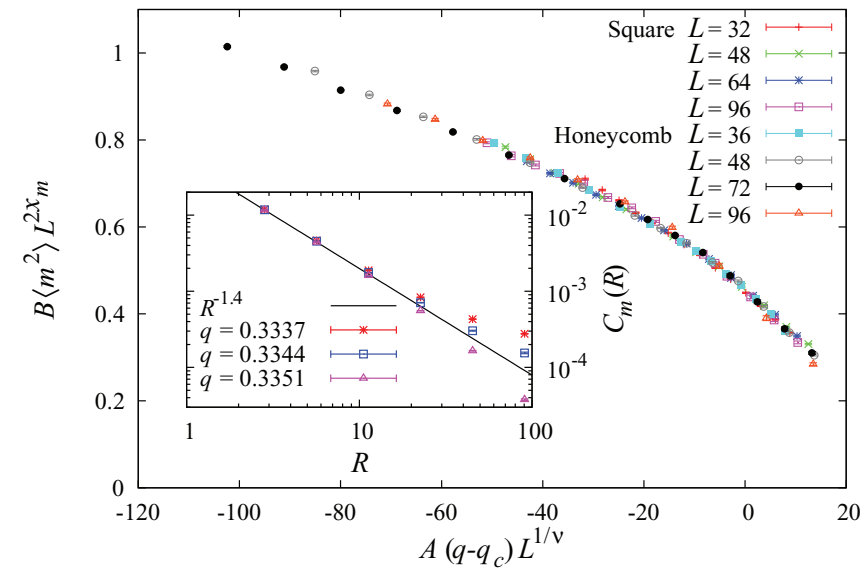

FIG. 2. (Color online) The FSS plot for the magnetization of the $\mathrm{SU}(3) J-Q$ model on the square and the honeycomb lattices with the system size being restricted to $L \leqslant 96$. The scaling factors $A=2.218$ and $B=1.752$ are multiplied to the horizontal axis and the vertical axis, respectively, for the results of the honeycomb lattice ( $A=B=1$ for the square lattice.) Inset: The two-point correlation function of magnetic order for the SU(3) models on a square lattice of $L=256$ as a function of the distance at various $q$ near the critical point. The straight line corresponds to the estimate of $2 x_{m}$ from the squared magnetization.

lattice of $L=256$. The critical value estimated from the data of $L \leqslant 256$ is $q=0.3343(1)$. While it is slightly smaller than the one quoted above for smaller systems, the correlation decay up to $R \sim 24$ lattice units is not so sensitive to $q$ and is well characterized by the exponent obtained above from the squared magnetization.

Scaling of the VBS order parameter. We define the local complex order parameter characterizing the VBS phase as $\Psi_{r} \equiv \sum_{\mu=1}^{z} e^{\frac{2 \pi i}{z} \mu} \hat{P}_{r, r_{\mu}}$ where $z$ is the coordination number of a lattice, $r_{\mu}$ is the neighboring site of $r$ in the direction $\mu$ [see Figs. 1(c) and 1(d)], and $\hat{P}_{r, r_{\mu}}$ is the diagonal part of the projection operator, respectively. According to Read and Sachdev, ${ }^{6,24}$ the complex VBS operator $\Psi$ corresponds to the annihilation operators of skyrmions, and it is then interpreted $^{2}$ in the continuum limit as $\Psi \sim \psi_{\uparrow} \psi_{\downarrow}^{*}$, where $\psi_{\alpha}$ is the meron (half-skyrmion) annihilation operator with an up $(\alpha=\uparrow)$ or down $(\alpha=\downarrow)$ spin at the core. In Fig. 3, the FSS of the squared amplitude of the total VBS order $\left\langle|\Psi|^{2}\right\rangle \equiv V^{-2}\left\langle\left|\sum_{r} \Psi_{r}\right|^{2}\right\rangle$ is presented for the SU(3) model. Similar to Fig. 2, we again restrict the system size to $L \leqslant 96$ and assume the same critical dimensions for both lattices. Namely, we assume $y=1.72$ and $2 x_{\Psi}=1+\eta_{\Psi}=1.47$ for both lattices while $q_{c}=0.3339$ and $q_{c}=0.2029$ for the square and honeycomb lattices, respectively. Note that the value of the scaling exponent $y$ is close (and actually consistent within the statistical error) to the estimate of $y$ based on the magnetization discussed above. From a similar analysis we obtain a reasonably good FSS plot for the SU(2) model when we assume $y=1.73$ and $2 x_{\Psi}=1.36$ for the scaling exponents and $q_{c}=0.9552$ and $q_{c}=0.5420$ for the square and honeycomb lattices, respectively. Note here that we again use data for $L \leqslant 128$ and 96 for the square and honeycomb lattices, respectively. As for the SU(4) model for $L \leqslant 96$, the best fitting is obtained with $y=1.40$ and $2 x_{\Psi}=1.73$ at 


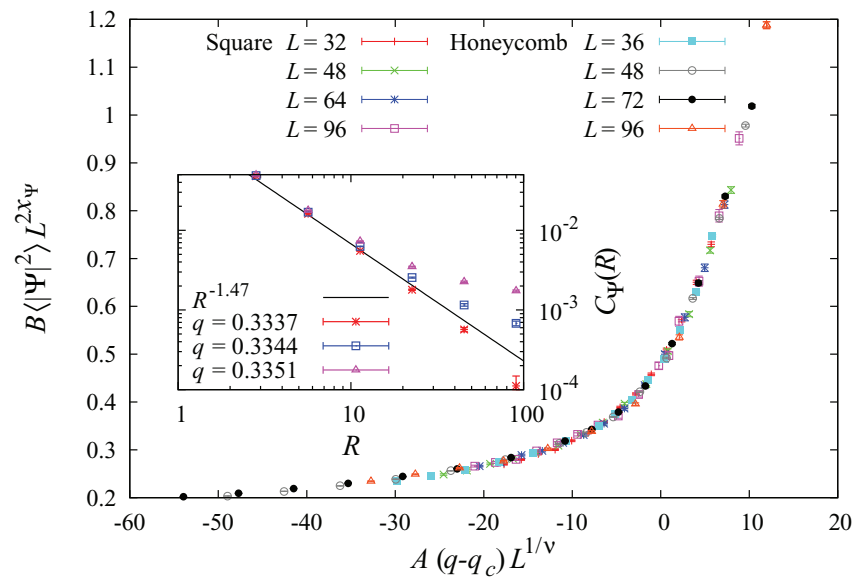

FIG. 3. (Color online) The FSS plot for the squared amplitude of total VBS order for the SU(3) $J-Q$ model on the square and the honeycomb lattices with the system size being restricted to $L \leqslant$ 96. We set the scaling factors $A=2.422$ and $B=0.6388$ for the honeycomb lattice. Inset: The two-point correlation function of VBS order for the SU(3) models on a square lattice of $L=256$ as a function of the distance at various $q$ near the critical point. The straight line corresponds to the estimate for $2 x_{\Psi}$ obtained from the squared amplitude of the total VBS order.

$q_{c}=0.0805$ and $q_{c}=0.0150$ for the square and the honeycomb lattice, respectively. The two-point correlator of the local VBS order parameter $C_{\Psi}\left(R_{i j}\right) \equiv\left\langle\Psi_{i}^{*} \Psi_{j}\right\rangle$ is also shown in the inset of Fig. 3. Again, we observe the consistency between the estimates of the critical indices obtained from $\left\langle|\Psi|^{2}\right\rangle$ and $C_{\Psi}$.

Systematic size dependence. The strongest skepticism concerning the critical nature of the phase transition comes from the argument ${ }^{16}$ that the true nature of the transition is revealed only in a very long range behavior, and that the previously attainable system size might not have reached that regime. In order to see the systematic trend as we go to a larger length scale, we apply the FSS analysis for quadruplets of the system sizes from $L_{\max } / 3$ to $L_{\max }$ such as $\{32,48,64$, $\left.96\left(=L_{\max }\right)\right\}$, and systematically change the value of $L_{\max }$. In Fig. 4, we plot the $L_{\max }$ dependence of these estimated scaling dimensions. We use the same value of $q_{c}$ and $y \equiv 1 / v$ for both the magnetization and the VBS order parameter. (This time we have lifted the restriction that $y$ should be independent of the lattice.) As is evident, for the SU(2) and the SU(3) models, there is a systematic trend of increasing $y$ as a function of $L_{\max }$. Whether it will eventually reach the value $y=d+1=3$, the value expected for the first-order transition, cannot be judged from the present data. We should note here that large values of $y$ do not necessarily suggest the first-order transition since the $1 / N$ expansion $^{25}$ also predicts large values for the DCP fixed point for small $N$. The systematic decrease in the effective values of the scaling dimensions $2 x_{m}$ and $2 x_{\Psi}$ is not as strong as $y$, while both of them should converge to zero if the transition is of the first order. The size dependence of the SU(4) model looks somehow different from those of the other cases. The systematic drifts in the critical indices are much weaker.

Higher power of local VBS order. We can directly estimate the order of the quantum VBS phase transition through the relevance or irrelevance of the higher power of the local VBS order parameter $\Psi^{q}$ with $q \geqslant 2$. In order for the quantum

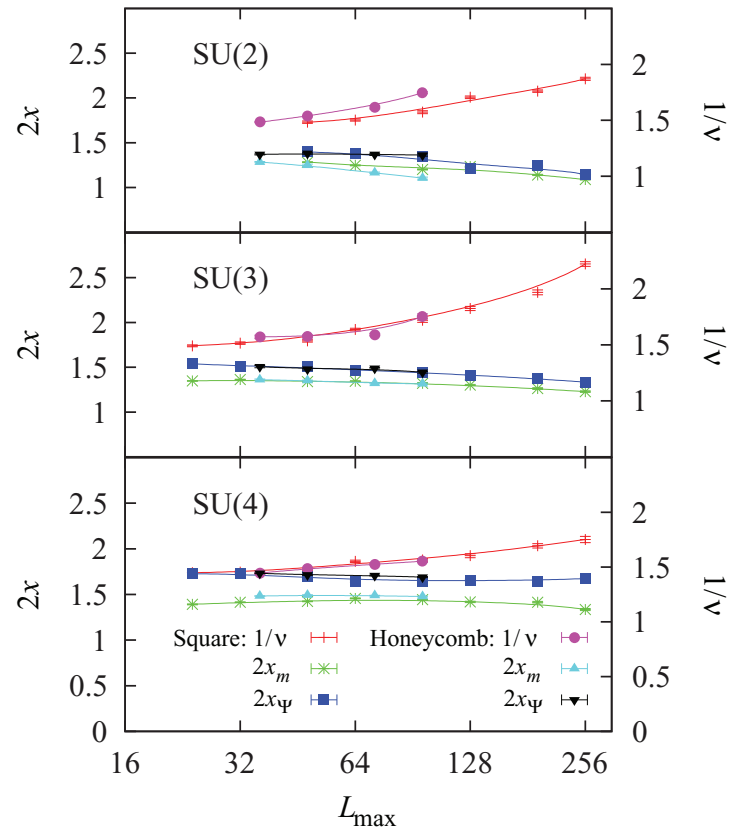

FIG. 4. (Color online) The estimate of the scaling dimensions $y \equiv 1 / v$ and $2 x$ for the $\mathrm{SU}(N)$ model on the square and honeycomb lattices as a function of the system size used in the FSS. At $L_{\max }=$ 256, $q_{c}=0.9568(2), 0.3343(1)$, and 0.0814(3) for $\mathrm{SU}(N=2,3,4)$ models on a square lattice, respectively.

transition on the square lattice to be of second order, $\Psi^{4}$ must be irrelevant since this field is naturally included in the Hamiltonian for the lattice with $Z_{4}$ symmetry. Similarly, the relevance or irrelevance of $\Psi^{3}$ and $\Psi^{2}$ is crucial, respectively, to the transition in the honeycomb lattice and to that in the square lattice with strong spatial anisotropy. ${ }^{26,27}$ The straightforward definition of $\Psi^{q}$ is not suitable here because of the discrete nature of the present $\Psi$ in contrast to its counterpart in the continuous field theory. Instead, we define the block-averaged order parameter $\bar{\Psi}_{i} \equiv \frac{1}{b^{d}} \sum_{j \in \Omega_{b}(i)} \Psi_{j}$, where $\Omega_{b}(i)$ is the region of a square with the linear scale $b$ centered at $i$. In Fig. 5, the two-point correlator for $q=2$, $C_{\Psi^{2}}\left(R_{i j}\right) \equiv\left\langle\left(\bar{\Psi}_{i}^{*}\right)^{2}\left(\bar{\Psi}_{j}\right)^{2}\right\rangle$ is plotted against the distance. From the figure, we estimate the scaling dimension of squared VBS order as $2 x_{\Psi^{2}} \sim 4.0$, or $y_{\Psi^{2}} \sim 1.0>0$, i.e., the skyrmion-pair operator is relevant at the deconfined critical point. This result shows that even if the DCP scenario is the correct

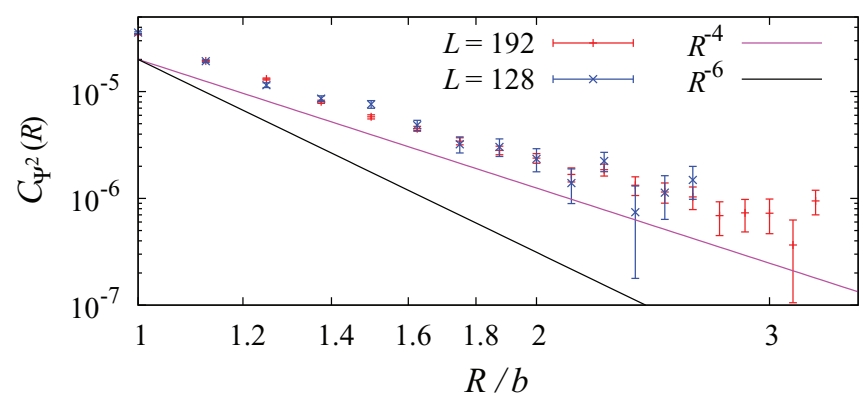

FIG. 5. (Color online) The two-point correlation function of the square of block-averaged order parameter $(\bar{\Psi})^{2}$ for the SU(3) models as a function of the distance at $q=0.3343$, which is the critical point estimated from the data of $L \leqslant 256$. The block size $b$ is 8 . 
description of the Néel-VBS quantum phase transition, it cannot take place in the $\mathrm{SU}(3) J-Q$ model with only $Z_{2}$ lattice rotational symmetry, where the model intrinsically contains the relevant perturbation $\Psi^{2}$. It follows, for example, that the quasi-one-dimensional biquadratic Heisenberg model should not have a DCP critical point. ${ }^{26}$ In previous work, ${ }^{26}$ the numerical results were analyzed mainly with the assumption of the criticality. This was partially due to the very slow onset of the order parameter as a function of the system size and the absence of a clear discontinuity, particularly for small systems. However, a trend similar to the one described above was already detected there; the large estimates of $y \equiv 1 / v$ [e.g., $y=2.5$ (2) for the VBS order parameter, and $y=2.9$ (2) for the magnetization order parameter]. The discontinuous nature of the spatially anisotropic $\mathrm{SU}(3)$ model is also consistent with the conclusion of a recent study based on the FSS analysis for the same model. ${ }^{28}$ In the cases of $q \geqslant 3$, the scaling dimension is probably larger than that of $q=2$. However, we have not succeeded in reliably estimating them due to relatively large statistical noises.

Conclusions. We have presented a series of numerical results on the $\mathrm{SU}(N) J-Q$ models on two lattices, square and honeycomb ones. Up to the system size explored in the present study, all the scaling analyses work fine as long as the range of the system size is not broad. Based on the assumption of the criticality, we have estimated various critical indices. The estimates obtained with different quantities on different lattices are consistent with each other. In addition, the agreement between the numerical estimates of the critical exponents $x_{\Psi}$ and $x_{m}$ with the $1 / N$ expansion $^{25}$ is still good even after the present updates of the former based on the largest systems. These pieces of evidence are consistent with the DCP scenario. However, the previous estimate of $y$ in Ref. 13 had to be considerably shifted beyond the error estimated then. This trend is systematic; for the $\mathrm{SU}(2)$ and $\mathrm{SU}(3)$ cases at least, the estimate increases as the system becomes larger and it seems to continue to grow beyond the largest estimate obtained in the present work. Because of these observations, we have to keep the possibility of a first-order transition still open. If the transition is of first order, the question will be about the reason for the apparent universal behaviors. It would be rather difficult to explain the observed behaviors unless the DCP fixed point exists close to the renormalization group trajectory of the present model, even if it may not be the governing fixed point. Whether the difference between $\mathrm{SU}(4)$ and the other two cases persists for larger systems is also an important question and requires further studies.

Acknowledgments. The computation in the present work was executed on computers at the Supercomputer Center, ISSP, University of Tokyo, and also on the K computer at the RIKEN AICS (Project No. hp120283). The present work is financially supported by MEXT KAKENHI No. 25287097, and by CMSI, MEXT-SPIRE, Japan.
${ }^{1}$ T. Senthil, A. Vishwanath, L. Balentz, S. Sachdev, and M. P. A. Fisher, Science 303, 1490 (2004).

${ }^{2}$ T. Senthil, L. Balents, S. Sachdev, A. Vishwanath, and M. P. A. Fisher, Phys. Rev. B 70, 144407 (2004).

${ }^{3}$ T. Senthil, L. Balents, S. Sachdev, A. Vishwanath, and M. P. A. Fisher, J. Phys. Soc. Jpn. Suppl. 74, 1 (2005).

${ }^{4}$ R. Darradi, O. Derzhko, R. Zinke, J. Schulenburg, S. E. Krüger, and J. Richter, Phys. Rev. B 78, 214415 (2008).

${ }^{5}$ R. K. Kaul, M. A. Metlitski, S. Sachdev, and C. Xu, Phys. Rev. B 78, 045110 (2008).

${ }^{6}$ N. Read and S. Sachdev, Phys. Rev. Lett. 62, 1694 (1989).

${ }^{7}$ K. Harada, M. Troyer, and N. Kawashima, J. Phys. Soc. Jpn. 67, 1130 (1998).

${ }^{8}$ K. Harada, N. Kawashima, and M. Troyer, Phys. Rev. Lett. 90, 117203 (2003).

${ }^{9}$ N. Kawashima and Y. Tanabe, Phys. Rev. Lett. 98, 057202 (2007).

${ }^{10}$ A. W. Sandvik, Phys. Rev. Lett. 98, 227202 (2007).

${ }^{11}$ R. G. Melko and R. K. Kaul, Phys. Rev. Lett. 100, 017203 (2008).

${ }^{12}$ A. W. Sandvik, Phys. Rev. Lett. 104, 177201 (2010).

${ }^{13}$ J. Lou, A. W. Sandvik, and N. Kawashima, Phys. Rev. B 80, 180414 (2009).

${ }^{14}$ R. K. Kaul and A. W. Sandvik, Phys. Rev. Lett. 108, 137201 (2012).

${ }^{15}$ R. K. Kaul, Phys. Rev. B 85, 180411(R) (2012).
${ }^{16}$ A. B. Kuklov, M. Matsumoto, N. V. Prokof'ev, B. V. Svistunov, and M. Troyer, Phys. Rev. Lett. 101, 050405 (2008).

${ }^{17}$ K. Chen, Y. Huang, Y. Deng, A. B. Kuklov, N. V. Prokof'ev, and B. V. Svistunov, Phys. Rev. Lett. 110, 185701 (2013).

${ }^{18}$ H. G. Evertz, G. Lana, and M. Marcu, Phys. Rev. Lett. 70, 875 (1993).

${ }^{19}$ S. Todo and K. Kato, Phys. Rev. Lett. 87, 047203 (2001).

${ }^{20}$ B. Bauer, L. D. Carr, H. G. Evertz, A. Feiguin, J. Freire, S. Fuchs, L. Gamper, J. Gukelberger, E. Gull, S. Guertler, A. Hehn, R. Igarashi, S. V. Isakov, D. Koop, P. N. Ma, P. Mates, H. Matsuo, O. Parcollet, G. Pawlowski, J. D. Picon, L. Pollet, E. Santos, V. W. Scarola, U. Schollwöck, C. Silva, B. Surer, S. Todo, S. Trebst, M. Troyer, M. L. Wall, P. Werner, and S. Wessel, J. Stat. Mech. (2011) P05001.

${ }^{21} \mathrm{~S}$. Todo, H. Matsuo, and H. Shitara (unpublished).

${ }^{22}$ K. Harada, Phys. Rev. E 84, 056704 (2011).

${ }^{23}$ S. Pujari, K. Damle, and F. Alet, Phys. Rev. Lett. 111, 087203 (2013).

${ }^{24}$ N. Read and S. Sachdev, Phys. Rev. B 42, 4568 (1990).

${ }^{25}$ R. K. Kaul and S. Sachdev, Phys. Rev. B 77, 155105 (2008).

${ }^{26}$ K. Harada, N. Kawashima, and M. Troyer, J. Phys. Soc. Jpn. 76, 013703 (2007)

${ }^{27}$ T. Grover and T. Senthil, Phys. Rev. Lett. 98, 247202 (2007).

${ }^{28}$ M. S. Block, R. G. Melko, and R. K. Kaul, Phys. Rev. Lett. 111, 137202 (2013). 